\title{
Giant cavernous hemangioma of the right atrium
}

\author{
Ivilin Todorov ${ }^{1}$, Zdravka P. Todorova ${ }^{2}$, and Dimitar P. Nikolov ${ }^{1}$ \\ ${ }^{1}$ Department of cardiothoracic surgery Acibadem City Clinic Tokuda Hospital Sofia \\ Bulgaria \\ ${ }^{2}$ Medicinski universitet-Sofia Medicinskii fakultet
}

December 17, 2021

\begin{abstract}
Cardiac hemangiomas as a primary heart tumor are extremely rare. We present a clinical case of a 54-year-old woman with atypical thoracic and abdominal discomfort and cavernous hemangiomas of the right atrium and the liver.
\end{abstract}

Title: Giant cavernous hemangioma of the right atrium

Running head: Right atrium cavernous hemangioma

Word counts: 1498

Authors:

Corresponding author: Ivilin P. Todorov, MD, ORCID ID 0000-0002-6556-4304

Affiliations: Department of cardiothoracic surgery

Acibadem City Clinic Tokuda Hospital, Sofia, Bulgaria

ivilint@gmail.com

Zdravka P. Todorova, MD, ORCID ID: 0000-0002-2969-5681

Affiliations: Department of Endocrinology, Specialized Hospital for Active Treatment of Pediatric Diseases, Faculty of Medicine, Medical University -Sofia, Sofia, Bulgaria

todorova.zdr@gmail.com

Dimitar P. Nikolov, MD, PhD

Affiliations : Department of cardiothoracic surgery

Acibadem City Clinic Tokuda Hospital, Sofia, Bulgaria

dpnikolov@abv.bg

"There are no prior publications or submissions with any overlapping information, including studies and patients."

Conflict of interest: None to declare

Funding: None to declare

Giant cavernous hemangioma of the right atrium

SUMMARY 
Cardiac hemangiomas as a primary heart tumor are extremely rare. We present a clinical case of a 54-yearold woman with atypical thoracic and abdominal discomfort and cavernous hemangiomas of the right atrium and the liver.

Key words: cardiac neoplasm, cavernous hemangioma, right atrium

\section{INTRODUCTION}

Cardiac hemangiomas are rare primary benign tumors that can occur at any age and are localized in any heart cavity.By the end of the 1980s, only 49 cases of cardiac hemangiomas had been reported in the literature ${ }^{[1]}$. About 250 cases have been described in the literature so far. Despite the benign nature, in the absence of radical removal of the tumor, the prognosis is poor. The most common causes of death in these patients are ventricular tachycardia, sudden cardiac death and local tumor progression ${ }^{[2]}$. Timely diagnosis and surgical treatment are extremely important in these patients.

\section{CLINICAL CASE}

IRB approval, consent statement and clinical trial registration are not applicable for this case report.

A 54-year-old woman was admitted to the clinic with complaints of retrosternal and epigastric pain with a duration of 4 months without a provocative moment against the background of well-controlled longterm arterial hypertension. Two years ago, selective coronary angiography (SCAG) was performed due to similar complaints, which did not show coronary heart disease. Ten years ago, the patient underwent a total hysterectomy followed by radiation therapy for uterine leiomyoma. One month before admission to our clinic, the patient was hospitalized in an abdominal surgery clinic with complaints of abdominal pain, nausea, single vomiting, and clinical evidence of ileus. Conducted exploratory laparoscopy with evidence of hemangioma of the left dome of the liver with dimensions $3 / 3.5 \mathrm{~cm}$ and a formation protruding along the right diaphragmatic dome, which after the release of ligamentum falciforme hepatitis, did not show to come from the liver. Referred to a cardiac surgery clinic to clarify the diagnosis. Upon admission to our clinic, the physical examination and chest X-ray showed no abnormalities. Laboratory tests are also normal. Computed axial tomography (CT) was performed with the result: heterodenous soft tissue formation with dimensions $82 / 67 / 78 \mathrm{~mm}$., With a pronounced "mass" effect on the superior vena cava and right atrium (RA). (Fig. 1). The formation contrasts centripetal, nodular and progressive in the three contrasting phases, with the hypodense center persisting in all phases. A rounded hypodense zone with dimensions of $22 / 21 \mathrm{~mm}$ in the area of the 2 nd hepatic segment is also described.

Nonspecific repolarization changes in the precordial leads are recorded on the ECG. Transthoracic echocardiography was performed, which established a structure that compresses the RA with a hypo-echogenic center (Fig. 1). Other echocardiographic parameters, including global and regional left ventricular function and valve function, are normal. There is no evidence of pericardial effusion. A preoperative SCAG was performed showing an abnormal blood supply to a paracardial tumor from a branch of the circumflex coronary artery in the absence of significant coronary stenosis (Fig. 3). Cardiac surgery was performed under C PB conditions and an encapsulated tumor formation with dimensions of $70 / 80 \mathrm{~mm}$, tightly fused with RA and free from the pericardium was found (Fig. 2). Due to the dense adhesion, no dissection of the formation was possible, and total excision was required together with the lateral wall of the RA to the mouths of the inferior and superior vena cava. The RA wall was restored using autologous pericardial patch plastic. The patient was extubated at 6 hours postoperatively with good respiratory mechanics. Drainages removed on the 2nd postoperative day. On the fifth postoperative day, due to persistent full AV-block, a permanent pacemaker SJM Endurity Core DDDR is implanted. Histological examination revealed a cavernous hemangioma composed of multiple dilated blood-filled lumens upholstered in a single epithelial epithelium and separated by hypocellular fibrous septa. The central part of the tumor is represented by a stroma with pronounced edema and mild chronic inflammatory infiltration. The patient was discharged on the 7 th postoperative day afebrile, hemodynamically stable, with primary healing surgical wounds, active, in very good general condition. At 18 months of follow-up, it remained asymptomatic, with no recurrence of the tumor formation. Recommendations are given for further follow-up. 


\section{Comment}

The incidence of primary cardiac tumors was very low, ranging from 0.002 to $0.3 \%$ in autopsy studies, with nearly $80 \%$ of them benign. Histologically, they are divided into cavernous, capillary, venous and mixed hemangiomas, of which $50 \%$ grow planar intramurally and $50 \%$ - exophytic intracavitary ${ }^{[3]}$. The incidence of hemangiomas among primary cardiac tumors in large operative series averages $1.4 \%{ }^{[4]}$. The right atrium is more often affected than the left ${ }^{[5]}$. Cases of combination with extracardiac hemangiomas in the gastrointestinal tract, skin or face have also been reported. They are extremely rare. In our case, it is a cavernous hemangioma in the RA, combined with a hepatic hemangioma. The clinical picture is very diverse and depends on the patient's age, location, size and growth rate of the tumor. It ranges from asymptomatic, to palpitations, arrhythmias, heart failure, pericardial effusion, pseudoangina, emboli, sudden cardiac death, and symptoms caused by compression of neighboring organs such as the trachea and esophagus. When localized in the atrial and interventricular septum, there is a high risk of sudden cardiac death ${ }^{[6]}$. Histological findings are essential for the final diagnosis. Many other imaging studies can provide additional information on some details. These are echocardiography, computed axial tomography (CT), coronary computed tomography, magnetic resonance imaging ${ }^{[7]}$. Echocardiography has certain limitations because it is unable to fully assess structures such as the mediastinum, diaphragm, and lung parenchyma that are difficult to interpret. Computed axial tomography (CT) provides more reliable information because it better demonstrates the location of the tumor and its connection with neighboring structures. The suspicion of hemangioma is high with abundant vascularization. SCAG may show a characteristic contrast finding - "tumor blush" [8] - fig.3. This was also observed in our case. The development of cardiac hemangiomas is unpredictable. They can spontaneously regress, become stationary or continue to grow ${ }^{[2]}$. Total surgical excision is the only definitive therapy. The prognosis is good with completely removed hemangiomas. Complete surgical resection results in complete cure, although rare cases of recurrence have been reported ${ }^{[6]}$. If radical resection is not possible, the prognosis is poor. Although there are no controlled studies, the use of corticosteroids, radiation therapy, beta blockers and vascular endothelial growth factor antagonists has been suggested in patients with unresectable tumors ${ }^{[4]}$. A multidisciplinary approach is extremely important in these patients.

\section{CONCLUSION}

Cardiac hemangiomas are rare, benign tumors of unknown etiology. There are currently no randomized trials and recommendations based on evidence of their behavior. Total surgical resection and removal of the tumor is the only definitive therapy in symptomatic patients.

Ivilin P. Todorov, MD- Concept/design, surgical intervention

Zdravka P. Todorova, MD- Drafting article, Data collection.

Dimitar P. Nikolov, MD, PhD - Critical revision of article, Approval of article.

\section{REFERENCES}

1.Konermann M, Sorge-Hädicke B, Grötz J, Josephs W, Hötzinger H. Hämangiom der linken Koronararterie mit Fistel in die Arteria pulmonalis.Dtsch. med. Wschr. 114: 1363-1366, 1989.

2. Van Trigt P III, Sabiston DC Jr. Tumors of the heart. In: SabistonDC Jr, Spencer FC, editors. Surgery of the chest. Vol 2. 6th ed.Philadelphia: WB Saunders, 1995:2069-88.

3.McAllister HA , Fenoglio JJJr. Tumors of the Cardiovascular System.Atlas of Tumor Pathology, Washington DC, Armed Forces Institute ofPathology - 2nd series, Vol. 15, p.253-254, 1978.

4.Miralles A, Bracamonte L, Soncul H, et. Al.: Cardiac tumors: clinical experience and surgical results in 74 patients. Ann. Thorac. Surg . 52:886-895, 1991.

5. Friedberg Ch. Tumoren des Herzens. In: Erkrankungen des Herzens.Hrsg. von Hegglin M., Aufl . Thieme Verlag, Stuttgart, Bd.II, Kap.46: 1972. 
6. Miao H, Yang W, Zhou M, Zhu Q, Jiang Z. Atrial hemangioma: a case report and review of the literature. Ann Thorac Cardiovasc Surg. 2019;25(2):7181.

7. Samanidis G, Khoury M, Balanika M, et al. Cardiac cavernous haemangioma: diagnosis and treatment. ANZ J Surg. 2014;84(4):2945.

8. Pigato JB, Subramanian VA, McCaba JC. Cardiac hemangioma.A case report and discussion. Tex Heart. Inst J. 1998;25:8385.

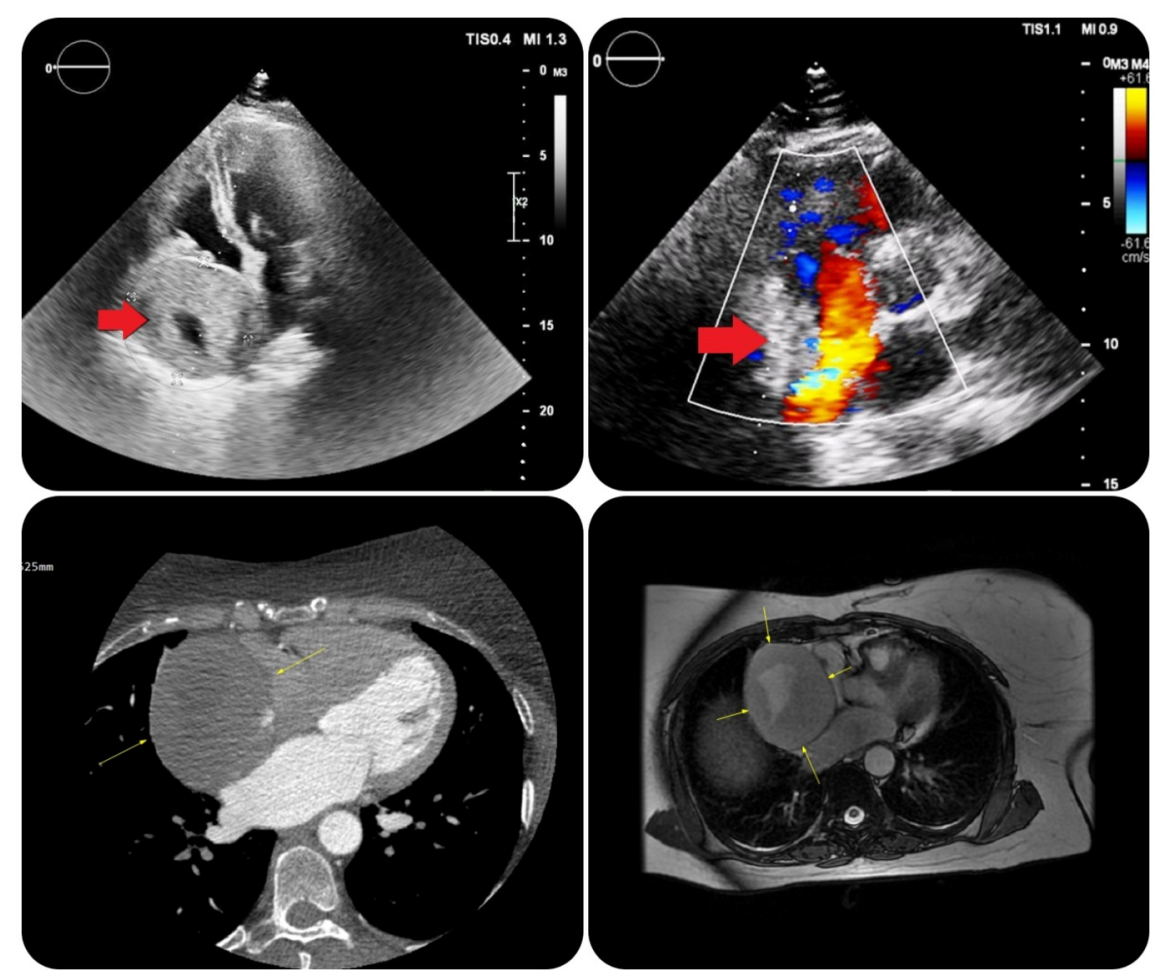

Fig.1: Echocardiographic, computed axial tomography (CT) and nuclear magnetic resonance (NMR) 


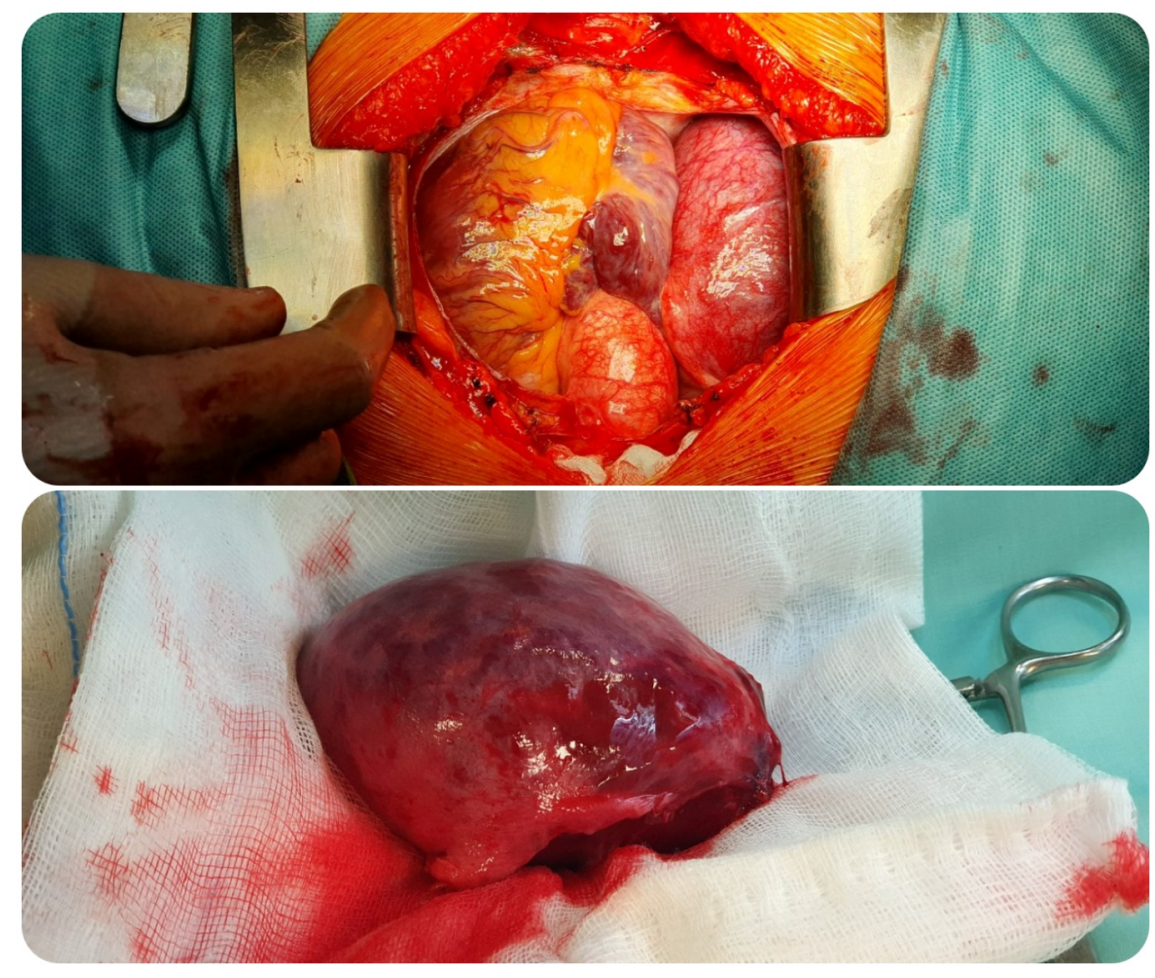

Fig.2: Intraoperative findings

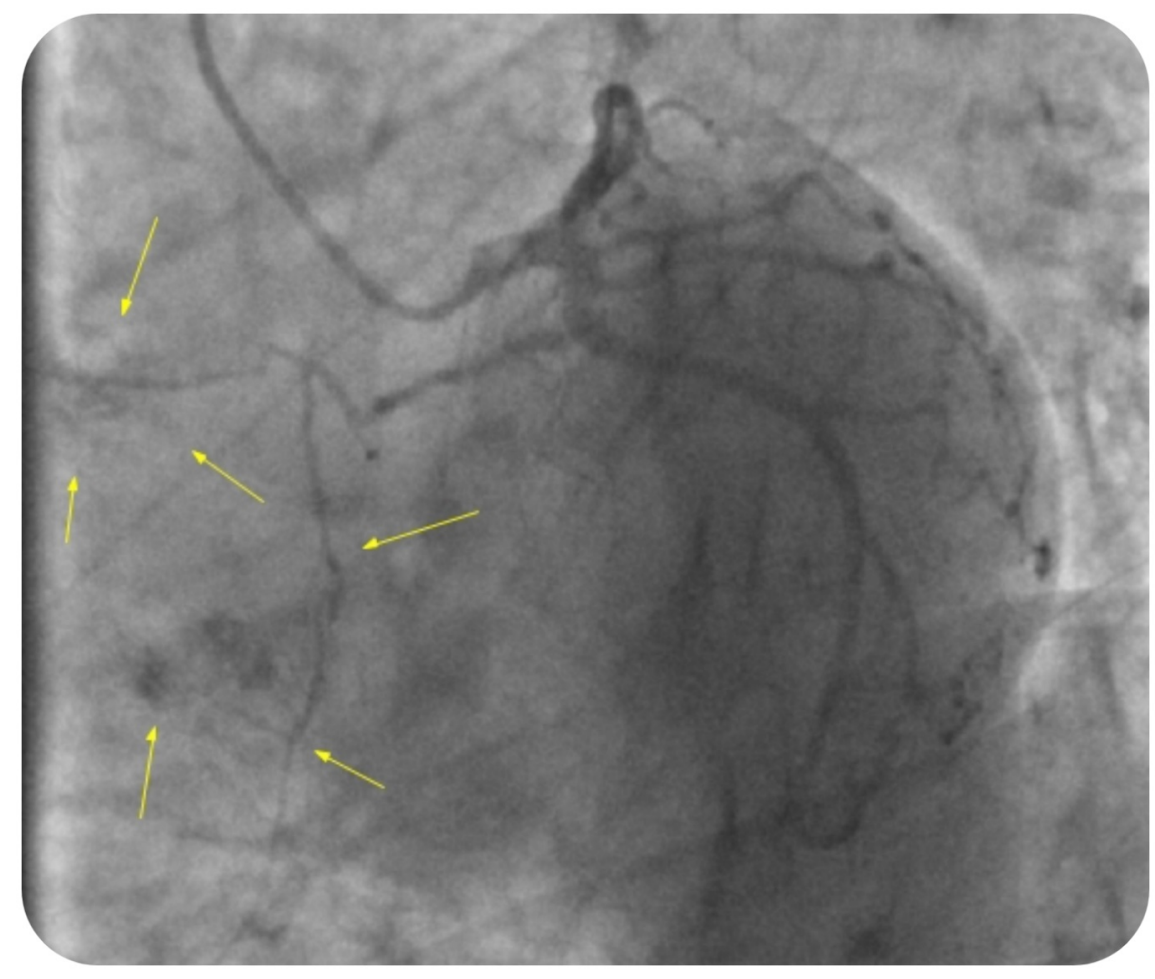


Fig3: SCAG show abnormal blood supply to a tumor from a branch of the circumflex coronary artery and very specific contrast finding - "tumor blush" 